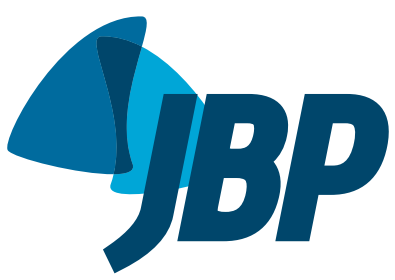

\section{Paravertebral mass}

\author{
Edson Marchiori1,a, Bruno Hochhegger ${ }^{2, b}$, Gláucia Zanetti1,c
}

A 59-year-old man presented with anemia and weight loss. He had a history of sickle-cell disease since he was 10 years of age. Chest CT scans revealed bilateral, well-marginated paravertebral masses in the lower half of the thorax, with heterogeneous density (Figure 1). He also presented with a large splenic calcification.

\section{DISCUSSION}

The CT features presented by the patient are typical of extramedullary hematopoiesis (EMH), defined as the development and growth of hematopoietic tissue outside the bone marrow. The main considerations in the differential diagnosis of masses in the posterior mediastinum, particularly in the paravertebral region, include neurogenic masses, lymphoma, paravertebral abscess, lateral meningocele, and $\mathrm{EMH}$. EMH is seen in a variety of hematologic disorders, particularly severe hemolytic anemia (thalassemia, sickle-cell anemia, and spherocytosis). Extensive replacement of normal bone marrow occurs when production is insufficient to meet the demands of the body. The most common sites of EMH are the liver, spleen, and lymph nodes, although it can occur in any organ. Thoracic involvement is less frequent, usually manifesting as bilateral lobulated masses in the lower paravertebral areas. The destruction of adjacent ribs and vertebrae is not seen in EMH. The erythropoietic masses are usually asymptomatic, although the presence of EMH within the spinal canal may be associated with spinal cord compression and neurologic deficit related to the level of involvement. The imaging manifestations of thoracic EMH can be unilateral or bilateral, sharply circumscribed, often lobulated, paraspinal soft-tissue masses, ${ }^{(1)}$ most frequently seen in the distal thoracic paraspinal region. CT scans can also be helpful in detecting areas of fat attenuation within these lesions, in depicting bony changes related to hematologic disorders, such as thalassemia and sickle-cell anemia, and in demonstrating splenic infarcts with focal calcifications or a small and dense calcified spleen (autosplenectomy), which can aid the differential diagnosis. The diagnosis of EMH can be established with reasonable certainty on the basis of characteristic radiologic findings in a patient with a predisposing hematologic condition. Invasive diagnostic procedures are potentially hazardous because of the highly vascular nature of the thoracic masses and the hemorrhagic potential of this condition. Microscopic examination shows well-formed hematopoietic tissue. In conclusion, the presence of bilateral, paravertebral masses with associated splenic calcifications are highly suggestive of $\mathrm{EMH}$. The main clinical finding in these patients is anemia.

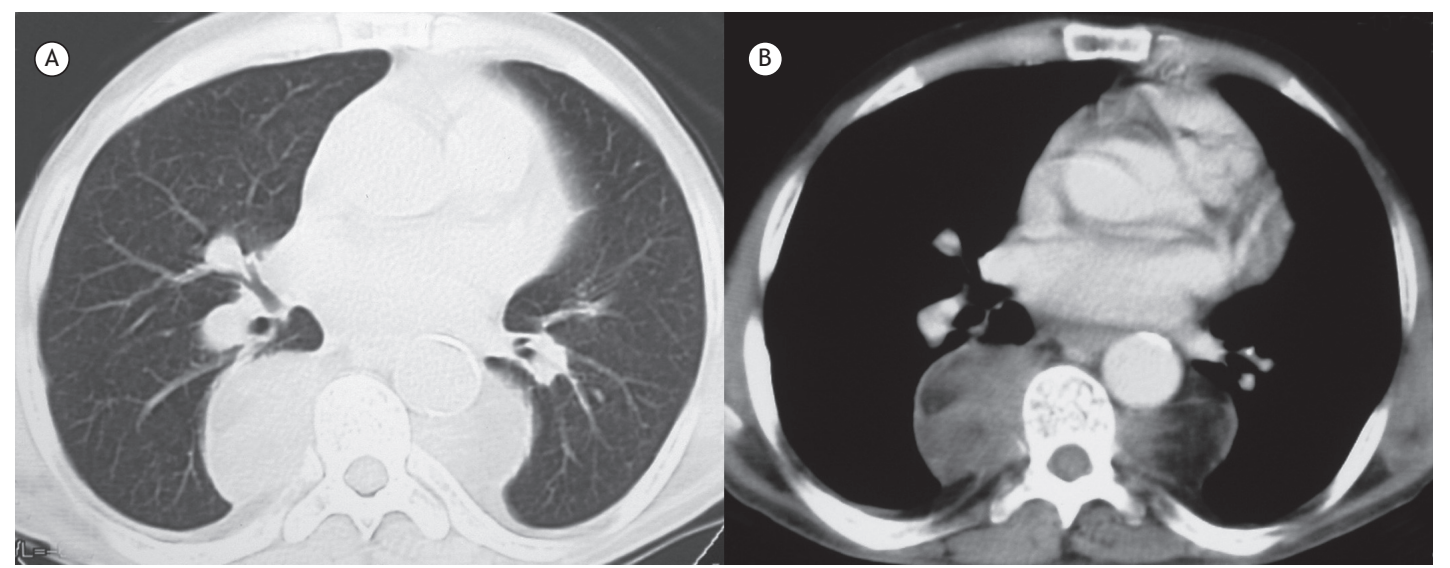

Figure 1. Chest CT scans with lung window (in A) and mediastinal window (in B) settings showing bilateral masses in the inferior paravertebral thoracic regions. The masses were heterogeneous, with low density areas, suggesting a fatty component. The lung parenchyma showed no abnormalities. There is also a large splenic calcification (not shown).

\title{
REFERENCE
}

Marchiori E, Escuissato DL, Irion KL, Zanetti G, Rodrigues RS, Meirelles GS, et al. Extramedullary hematopoiesis: findings on computed 